PROCEEDINGS OF THE

AMERICAN MATHEMATICAL SOCIETY

Volume 128, Number 4, Pages 1247-1256

S 0002-9939(99)05162-X

Article electronically published on July 8, 1999

\title{
SMALL INDUCTIVE DIMENSION OF COMPLETIONS OF METRIC SPACES. II
}

\author{
S. MRÓWKA
}

(Communicated by Alan Dow)

\begin{abstract}
Extending the results of a previous paper under the same title we show that, under $\mathbf{S}\left(\aleph_{0}\right)$, ind ${ }_{c} \nu \mu_{0}^{2}=2$.
\end{abstract}

\section{INTRODUCTION}

In this paper we continue the study of the small inductive dimension, ind, of completions of esoteric spaces $(=$ metric spaces with ind $\neq \operatorname{dim})$ initiated in a previous paper under the same title [M2]. In [M2] we have described the metric space $\nu \mu_{0}$ such that ind $\nu \mu_{0}=0$, but, under the following condition every completion of $\nu \mu_{0}$ contains an interval and therefore ind ${ }_{c} \nu \mu_{0}=1$, where, for a metric space $M$, ind ${ }_{c} M$ stands for $\min \{$ ind $\tilde{M}: \tilde{M}$ is a completion of $M\}$ :

$\mathbf{S}\left(\aleph_{0}\right)$ : If $A$ is a set of cardinality $2^{\aleph_{0}}$, then the product $A^{\aleph_{0}}$ cannot be written as $A^{\aleph_{0}}=F_{1} \cup F_{2} \cup \ldots$, where each $F_{n}$ is an $\mathbf{F}_{\sigma}$-set in the product topology of $A^{\aleph_{0}}$ (A - discrete) and it is countable on all lines parallel to the $n$-th axis 1

$\mathbf{S}\left(\aleph_{0}\right)$ disagrees very strongly with the continuum hypothesis, but fortunately its consistency with ZFC has been recently shown by Dougherty [Dou, who, in this way, terminated a long and unenviable period during which I was at the mercy of the logicians. In this paper we give a very natural continuation of [M2]; our main result is

1.1. Main Theorem. Under $\mathbf{S}\left(\aleph_{0}\right)$, every completion of $\nu \mu_{0}^{2}$ contains the square $[0,1]^{2}$ and therefore ind ${ }_{c} \nu \mu_{0}^{2}=\operatorname{dim} \nu \mu_{0}^{2}=2$.

The result concerning ind ${ }_{c} \nu \mu_{0}^{2}$ appears to be very natural. The problem of the equality ind $c_{c}=$ ind (which, for spaces with ind $=0$, can be stated without any reference to dimension theory: does every metric space having a base consisting of clopen sets have a completion with such a base?) is one of the simplest and most

Received by the editors March 9, 1998 and, in revised form, June 4, 1998.

1991 Mathematics Subject Classification. Primary 54F45; Secondary 54A35, 54E35, 54H05.

Key words and phrases. Inductive and covering dimension, metric spaces, completion, Bernstein sets, scattered sets.

${ }^{1}$ If $\tilde{a}$ is an element of the product $\prod_{\eta \in H} A_{\eta}$ and $\xi \in H$, then the line passing through $\tilde{a}$ and parallel to the $\xi$-th axis, $L_{\xi}^{\tilde{a}}$, is the set of all elements of $\prod_{\eta} A_{\eta}$ which differ from $\tilde{a}$ only in the $\xi$-th coordinate. We say that a subset $F$ of $\prod_{\eta} A_{\eta}$ has a property $\mathbf{P}$ on all lines parallel to the $\xi$-th axis provided that, for every $\tilde{a} \in \prod_{\eta} A_{\eta}, F \cap L_{\xi}^{\tilde{a}}$ has $\mathbf{P}$. More generally, if $\Xi \subset H$, then we can define hyperplanes parallel to the $\Xi$-th hyperaxis and speak about a set having a given property on all such hyperplanes. 
basic questions concerning concepts that were in existence for over 75 years. Before M2, nothing was known about the answer to this question (for non-separable spaces). Now the first step has been made; but only partial - not validity, only consistency. This is not unusual; it happens quite often that consistency results are obtained first because they are easier - or much easier - than validity or because validity cannot be attained at all and the final solution is the independence. But once the first step has been made, the next is following very quickly. In fact, as far as consistency is concerned, all the main questions concerning ind ${ }_{c}$ (or even $\operatorname{ind}_{c}^{*}(M)=\min \{\operatorname{ind}(\tilde{M} \backslash M): \tilde{M}$ is a completion of $M\}$; see part b) of 4.3) are now answered. However, the matters become more unusual if we look at dim. This definitely is not the first step in this direction; indeed, the first step, existence of metric spaces with $\mathrm{d}$-spread $=\operatorname{dim}-$ ind $=1$, had been made about 35 years ago $[\mathrm{R}$. So far all the efforts to raise the value of d-spread from 1 to 2 have failed. Now, in a way, this has been done but not as well as for d-spread $=1$. The reason why there is such a chasm between the first two values of d-spread is not yet understood.

A further consequence of Theorem 1.1 is the existence, in ZFC, of metric spaces for which dim cannot be determined in ZFC; indeed, under the continuum hypothesis, $\operatorname{dim} \nu \mu_{0}^{2}=1$ (the proof from [M3] applies). Concerning ind ${ }_{c}$, this was already achieved in [M2]: ind ${ }_{c} \nu \mu_{0}$ cannot be determined in ZFC (but note that, in ZFC, $\left.\operatorname{dim} \nu \mu_{0}=1\right)$. Since the existence of spaces with undeterminable dim and/or ind $c$ is an entirely new phenomenon, worthy of further development, an additional discussion of these matters is given in the problem section of this paper (sect. 5); here we will summarize what is known at the present moment:

1.2. The values of ind, ind ${ }_{c}$, and $\operatorname{dim}$ of $\nu \mu_{0}$ and $\nu \mu_{0}^{2}$ are as follows:

(i) $\quad$ in $Z F C: \quad$ ind $\nu \mu_{0}=\operatorname{ind} \nu \mu_{0}^{2}=0 ; \quad \operatorname{dim} \nu \mu_{0}=1$;

(ii) $\quad$ under $C H$ : $\quad \operatorname{ind}_{c} \nu \mu_{0}=\operatorname{ind}_{c} \nu \mu_{0}^{2}=0 ; \quad \operatorname{dim} \nu \mu_{0}^{2}=1$;

(iii) $\quad$ under $S\left(\aleph_{0}\right): \quad \operatorname{ind}_{c} \nu \mu_{0}=1 ; \quad \quad \operatorname{ind}_{c} \nu \mu_{0}^{2}=\operatorname{dim} \nu \mu_{0}^{2}=2$.

To keep the paper short we just show what is new rather than try to get the strongest version of the results; e.g., we work only with $\nu \mu_{0}^{2}$ although it is reasonably certain that better results could be obtained through the use of higher powers of $\nu \mu_{0}$.

Note. The author is pleased to report substantial progress in the area of esoteric spaces due to Kulesza [Ku1, $\mathrm{Ku} 2$ and also to Ostaszewski [Ost]. Kulesza has produced numerous modifications of $\Delta$ and $\mu_{0}$ and in this way he was able to vary proprieties of esoteric spaces to a large degree. In particular, he has a complete $\mathcal{N}$-compact esoteric space. He also studies irreducible esoteric spaces - in the sense that they do not contain esoteric subspaces of weight smaller than that of the space itself. This seems to be a promising direction of investigations; indeed, the concept of irreducibility can modified in many ways and this leads to a number of very natural but probably very difficult problems. Again, further comments on this matter are in sect. 5 .

Notation. Let $t$ be a number from $[0,1]$. If $t$ can be written in the form $t=$ $(2 k+1) 2^{-n}$, where $k$ is an integer and $n$ is a non-negative integer, then ( $t$ is called a dyadic rational and) we let ord $t=n$; if $t$ is a dyadic irrational, then, obviously, we let ord $t=\infty$. The letters $\mathcal{R}, \mathcal{N}, \mathcal{J}, \mathcal{P}, \mathcal{C}$ will stand, respectively, for the space 
of the reals, the positive integers, the closed interval $[0,1]$, the dyadic irrationals in $[0,1]$ and the Cantor set.

Concluding this introduction I wish to express my sincere gratitude to the referee for suggesting several changes, correcting several errors and for completing the report in a remarkably short time.

\section{TOOLS}

The only item of substance in this section is 2.5 . In addition, a variation of the concept of Bernstein sets (2.4 and above) is of interest.

A. $\sigma$-non-archimedean bases. A $\sigma$-non-archimedean base $\mathfrak{B}$ in a space $X$ is a sequence $\mathfrak{B}^{(1)}, \mathfrak{B}^{(2)}, \ldots$ such that each $\mathfrak{B}^{(k)}$ covers $X, U \in \mathfrak{B}^{(k)}, V \in \mathfrak{B}^{(j)}$ and $U \cap V \neq \emptyset$ imply $V \subset U$ for every $k, j=1,2, \ldots, k \leq j$ and $\bigcup_{k} \mathfrak{B}^{(k i)}$ is a base for $X$. We shall frequently identify $\mathfrak{B}$ with $\bigcup_{k} \mathfrak{B}^{(k)}$ (i.e., we will write $\mathfrak{B}=\bigcup_{k} \mathfrak{B}^{(k)}$ ). Note that the members $\mathfrak{B}^{(k)}$ are mutually disjoint, hence $\mathfrak{B}^{(k)}$ is a discrete collection of clopen sets. $\mathfrak{B}$ is called complete provided that for every descending sequence $U_{n}$ with $\emptyset \neq U_{n} \in \mathfrak{B}^{\left(i_{n}\right)}$, where $i_{n} \rightarrow \infty$, we have $\bigcap_{n} U_{n} \neq \emptyset$.

The following can be regarded as known.

2.1. A space $X$ has a $\sigma$-non-archimedean base iff it is strongly 0-dimensional and metrizable. A space $X$ has a complete $\sigma$-non-archimedean base iff it is strongly 0 -dimensional and metrizable in a complete way.

Historical note. The second sentence of 2.1 follows from a strongly 0-dimensional version of a result of Kuratowski:

2.2. If $X$ is a metric space with $\operatorname{dim} X=0$, then for every $\mathbf{G}_{\delta}$-subset $G$ of $X$ we have $G \underset{\mathrm{cl}}{\subset} X \times N^{\aleph_{0}}$.

The original Kuratowski result asserts: for every $\mathbf{G}_{\delta}$-subset $G$ of a metric space $X$ we have $G \underset{\mathrm{cl}}{\subset} X \times \mathcal{R}^{\aleph_{0}}$. Both versions are particular cases of 2.1 in [M1] (the Embedding Theorem) or - vice versa - the Embedding Theorem can be viewed as a natural continuation of the Kuratowski result. In turn, the Kuratowski result is a natural continuation of a very early result of Kuratowski and Sierpiński: if a subset $L$ of a metric space $X$ is the difference of two closed sets, then $L \underset{\mathrm{cl}}{\subset} X \times \mathcal{R}$. As far as I know, the above is the first result on closed embedding into products. If the reader knows earlier results, I would appreciate a note to this effect.

B. Bernstein sets. A Bernstein subset of a space $X$ is a set $S \subset X$ which intersects every non-empty perfect subset of $X$. It is known that Bernstein subsets of $\mathcal{J}^{2}$ are connected.

It is possible that the following is well-known.

2.3. If $S$ is a subset of $\mathcal{J}^{2}$ so that $S$ and $\mathcal{J}^{2} \backslash S$ are both Bernstein, then for every $\mathbf{G}_{\delta}$ set $G$ with $S \subset G \subset \mathcal{J}^{2}, G \backslash S$ is also Bernstein. Consequently, for every completion $\tilde{S}$ of $S, \tilde{S} \backslash S$ is connected.

Proof. Let $C \subset \mathcal{J}^{2}$ be a perfect set. Then $S \cap C$ is dense in $C$; thus $G \cap C$ is dense in $C$. Consequently, $G \cap C$ contains a perfect set $C_{1}$. Now, J $\mathrm{J}^{2} \backslash S$ intersects $C_{1}$; thus $G$ intersects $\mathcal{J}^{2} \backslash S$, and hence $G \backslash S$ intersects $C_{1}$. 
A subset $K$ of a product $X \times Y$ will be called oblique provided that for every $\left(x_{1}, y_{1}\right),\left(x_{2}, y_{2}\right) \in K,\left(x_{1}, y_{1}\right) \neq\left(x_{2}, y_{2}\right)$ imply $x_{1} \neq x_{2}$ and $y_{1} \neq y_{2}$; i.e., both projection maps of $K$ are one-to-one. A 2-Bernstein subset of a space $X$ is a set $S \subset X$ such that $S^{2}$ intersects every non-empty oblique perfect subset of $X^{2}$. The standard construction of disjoint Bernstein sets (in perfect metric spaces) can be easily adapted to yield that the Cantor set $\mathcal{C}$ contains two disjoint 2-Bernstein sets.

For one of the results in sect. 4 we need a further refinement. Let $\mathcal{D}$ be a collection of subsets of $Y$. A subset $S$ of $X$ is called $\mathcal{D}$-Bernstein provided that for every $D \in \mathcal{D}, S \times D$ intersects every non-empty oblique perfect subset of $X \times Y$. We have

2.4. If $X$ and $Y$ are complete dense-in-itself separable metric spaces and $\mathcal{D}$ is a collection of Bernstein subsets of $Y$ with card $\mathcal{D} \leq 2^{\aleph_{0}}$, then $X$ contains two disjoint $\mathcal{D}$-2-Bernstein sets $B^{(1)}$ and $B^{(2)}$.

A $\mathcal{D}$-2-Bernstein set is, obviously, a set which is 2 -Bernstein and $\mathcal{D}$-Bernstein. The above is not the best result: the number of $\mathcal{D}$-2-Bernstein sets can be increased and the result can be extended to $\mathcal{D}$ - $n$-Bernstein sets. But we need even less than 2.4 .

C. Scattered sets. Let $\mathbf{Q}$ be downward monotone property of closed sets (i.e., $F$ has $\mathbf{Q}$ and $F^{\prime} \subset F$ imply $F$ has $\mathbf{Q}$ ). We say that $F$ is $\mathbf{Q}$-scattered provided that for every non-empty closed $F^{\prime} \subset F$ there is an open $U$ so that $U \cap F^{\prime}$ is closed, non-empty and has $\mathbf{Q}$.

2.5. Let $X$ be a space with a $\sigma$-non-archimedean base $\mathfrak{B}=\mathfrak{B}^{(1)} \cup \mathfrak{B}^{(2)} \cup \ldots$ Let $\mathbf{Q}_{1}, \ldots, \mathbf{Q}_{l}$ be downward monotone properties of closed sets. If a closed set $F$ is $\mathbf{Q}_{1} \vee \cdots \vee \mathbf{Q}_{l}$-scattered, then there are closed sets $F_{k, i}$ and classes $\mathfrak{F}_{k, i} \subset \mathfrak{B}^{(k)}$, $k=1,2, \ldots, i=1, \ldots, l$, such that (i) $F=\bigcup_{k, i} F_{k, i}$, (ii) $F_{k, i} \subset \bigcup \mathfrak{F}_{k, i}$ and (iii) for every $U \in \mathfrak{F}_{k, i}, U \cap F_{k, i}$ has $\mathbf{Q}_{i}$.

Proof. Let $\lambda$ be an ordinal with $\bar{\lambda}>$ card $F$. We shall show that there exists a $\xi_{0}$ with $0<\xi_{0}<\lambda$ and such that for every $\eta<\xi_{0}$ we can define $F^{(\eta)}$ and $\mathfrak{F}_{k, i}^{(\eta)} \subset \mathfrak{B}^{(k)}$ so that (a) $F^{(\eta)}$ form a strictly decreasing sequence of closed sets, (b) $F^{(\eta)} \cap \bigcup\left\{\bigcup \mathfrak{F}_{k, i}^{\left(\eta^{\prime}\right)}: \eta^{\prime}<\eta, k=1,2, \ldots, i=1, \ldots, l\right\}=\emptyset$, (c) for every $U \in \mathfrak{F}_{k, i}^{(\eta)}$, $U \cap F^{(\eta)}$ has $\mathbf{Q}_{i}$, and $(\mathrm{d}) \bigcap_{\eta<\xi_{0}} F^{(\eta)}=\emptyset$.

Let $F^{(0)}=F$ and $\mathfrak{F}_{k, i}^{(0)}=\emptyset$. Assume that for some $\xi, 0<\xi<\lambda, F^{(\eta)}$ and $\mathfrak{F}_{k, i}^{(\eta)}$ are already defined for every $\eta<\xi$ so that (a), (b) and (c) are satisfied (this was so for $\xi=1$ ). If $\bigcap_{\eta<\xi} F^{(\eta)}=\emptyset$, then we terminate the induction. Otherwise, we continue letting $F^{(\xi)}=\bigcap_{\eta<\xi} F^{(\eta)} \backslash \bigcup\left\{\bigcup \mathfrak{F}_{k, i}^{(\eta)}: \eta<\xi, k=1,2, \ldots, i=1, \ldots, l\right\}$ and $\mathfrak{F}_{k, i}^{(\xi)}=\left\{U \in \mathfrak{B}^{(k)}: U \cap F^{(\xi)} \neq \emptyset, U \cap F^{(\xi)}\right.$ has $\left.\mathbf{Q}_{i}\right\}$ (if there are several $i$ 's so that $U \cap F^{(\xi)}$ has $\mathbf{Q}_{i}$, then use just one of them). Since $\bar{\lambda}>\operatorname{card} F$, the induction must terminate before reaching $\lambda$; i.e., the $\xi_{0}<\lambda$ exists. Observe that for $\xi<\xi^{\prime}<$ $\xi_{0}, \mathfrak{F}_{k, i}^{(\xi)}$ and $\mathfrak{F}_{k, i}^{\left(\xi^{\prime}\right)}$ are disjoint, indeed, $U \in \mathfrak{F}_{k, i}^{\left(\xi^{\prime}\right)}$ implies $U \cap F^{\left(\xi^{\prime}\right)} \neq \emptyset$ and $F^{\left(\xi^{\prime}\right)}$ is disjoint from $\bigcup \mathfrak{F}_{k, i}^{(\xi)}$. It follows that letting $\mathfrak{F}_{k, i}=\bigcup\left\{\mathfrak{F}_{k, i}^{(\eta)}: \eta<\xi_{0}\right\}$, we have, for a $U \in \mathfrak{F}_{k, i}, U \cap F=U \cap F^{\eta}$ for the unique $\eta$ with $U \in \mathfrak{F}_{k, i}^{(\eta)}$. Thus, letting $F_{k, i}=F \cap \bigcup \mathfrak{F}_{k, i}$ we complete the proof. 


\section{Proof OF THE MAIN THEOREM}

The proof of 1.1 will come after a sequence of intermediate results. We start with the description of objects used in the proof.

Recall that the construction of $\nu \mu$ and $\nu \mu_{0}$ involved the Cantor set $\mathcal{C}$ and its subset $A$. As in [M2] we need that card $A=2^{\aleph_{0}}$ but this time we also need that $B=\mathrm{C} \backslash A$ is 2-Bernstein. In addition, for 4.1 (the case concerning Bernstein set) we will need $B$ to be $\mathcal{D}$-2-Bernstein, where $\mathcal{D}$ will be specified in the proof of 4.1. Infinite sequences of elements of $\mathcal{C}$ (i.e., members of $\mathcal{C}^{\aleph_{0}}$ ) will be denoted as $\tilde{x}, \tilde{y}, \tilde{z}, \ldots$, possibly with subscripts, and furthermore $\tilde{x}(n)$ (and not $\tilde{x}_{n}$ ) will denote the $n$-th term of $\tilde{x}$. The letter $\mathfrak{a}$ will be reserved for members of $A^{\aleph_{0}}$; it will be used mainly in the case when such a member is built up by extracting terms from an $\tilde{x} \in \mathcal{C}^{\aleph_{0}}$ (see the definition of the maps $\Phi^{m_{1}, m_{2}}$ and $\Phi_{i}^{m}$ below). In the set $\left(A^{\aleph_{0}}\right)^{2}$ of all pairs $\left(\mathfrak{a}_{1}, \mathfrak{a}_{2}\right), \mathfrak{a}_{i} \in A^{\aleph_{0}} ; \mathfrak{a}_{i}(m)$ will be called the $(m, i)$-coordinate of $\left(\mathfrak{a}_{1}, \mathfrak{a}_{2}\right)$.

We need some maps. The map $\Phi^{m_{1}, m_{2}}:\left(A^{\aleph_{0}}\right)^{2} \rightarrow A^{\aleph_{0}} \times \mathcal{C} \times \mathcal{C}$ carries $\left(\tilde{x}_{1}, \tilde{x}_{2}\right)$ onto the triple $\left(\mathfrak{a}, c_{1}, c_{2}\right)$, where $\mathfrak{a} \in A^{\aleph_{0}}$ is a sequence built out of terms of $\tilde{x}_{1}$ and $\tilde{x}_{2}$, omitting the terms $\tilde{x}_{1}\left(m_{1}\right)$ and $\tilde{x}_{2}\left(m_{2}\right)$ and $c_{i}=\tilde{x}_{i}\left(m_{i}\right)$. The map $\Phi_{i}^{m}$ : $\left(A^{\aleph_{0}}\right)^{2} \rightarrow A^{\aleph_{0}} \times \mathcal{C}$ carries an $\left(\tilde{x}_{1}, \tilde{x}_{2}\right) \in\left(A^{\aleph_{0}}\right)^{2}$ onto the pair $(\mathfrak{a}, c)$, where $\mathfrak{a}$ is the sequence build out of terms of $\tilde{x}_{1}$ and $\tilde{x}_{2}$, omitting the term $\tilde{x}_{i}(m)$ and $c$ is $\tilde{x}_{i}(m)$. $\Phi^{m_{1}, m_{2}}$ and $\Phi_{i}^{m}$ are continuous and one-to-one.

Recall that the set of points of $\nu \mu$ is the set of all pairs $(\tilde{x}, t)$ where $t \in \mathcal{J}$ and $\tilde{x}(n) \in A$ for every $n \neq$ ord $t$. The topology is defined by the neighborhoods $U_{n}(\tilde{x}, t)$ :

$$
U_{n}(\tilde{x}, t)=\left\{(\tilde{y}, s): s \in I_{n}(t) \text { and } \tilde{y}(i)=\tilde{x}(i) \text { for } i \leq n\right\},
$$

if $n<$ ord $t$, and

$$
\begin{aligned}
U_{n}(\tilde{x}, t)= & \left\{(\tilde{y}, s): s \in I_{n}(t)\right. \text { and } \\
& \tilde{y}(i)=\tilde{x}(i) \text { for } i \leq n+1, i \neq \text { ord } t ; \tilde{y}(i)|n=\tilde{x}(i)| n \text { for } i=\text { ord } t\},
\end{aligned}
$$

if $n \geq$ ord $t$.

In the above, $I_{n}(t)$ is an open interval $(a, b)$ containing $t$ and so that $a$ and $b$ are the dyadic rationals of the smallest possible orders with $b-a=2^{-n} \cdot \nu \mu_{0}$ consists of all those $(\tilde{x}, t) \in \nu \mu$ for which $\tilde{x}(n) \in B$ for $n=\operatorname{ord} t$.

For $\tilde{x}, \tilde{y} \in \mathfrak{C}^{\aleph_{0}}$ the $\tilde{x} \tilde{y}$-plank (or a horizontal plank) of $\nu \mu^{2}$ is the set $H_{\tilde{x}, \tilde{y}}$ of all the $((\tilde{x}, t),(\tilde{y}, s)) \in \nu \mu^{2}$ with the fixed $\tilde{x}, \tilde{y}$. For a subspace $\kappa$ of $\nu \mu$, the $\tilde{x} \tilde{y}$-plank of $\kappa$ is the set $H_{\tilde{x}, \tilde{y}} \cap \kappa$. Let $\hat{h}=h \times h$ where $h: \nu \mu \rightarrow I$ is defined by $h(\tilde{x}, t)=t$ (i.e., $h$ is the function from 2.3 in [M2]). By 2.3 in [M2], $\hat{h}$ is a homeomorphism on each of the horizontal planks.

Let $t, t_{1}, t_{2}$ be dyadic rationals of orders $m, m_{1}, m_{2}$, respectively; we let $\mathcal{P}_{1}^{t}=$ $\{t\} \times \mathcal{P}, \mathcal{P}_{2}^{t}=\mathcal{P} \times\{t\}(\mathcal{P}$ is the set of all dyadic irrationals in $\mathcal{J}), \mathbb{P}^{t_{1}, t_{2}}=\hat{h}^{-1}\left(t_{1}, t_{2}\right)$, $\mathbb{P}_{i}^{t}=\hat{h}^{-1}\left[\mathcal{P}_{i}^{t}\right]$.

The map

$$
\hat{\Phi}^{t_{1}, t_{2}}\left(\left(\tilde{x}_{1}, t_{1}\right),\left(\tilde{x}_{2}, t_{2}\right)\right)=\Phi^{m_{1}, m_{2}}\left(\tilde{x}_{1}, \tilde{x}_{2}\right)
$$

is a homeomorphism of $\mathbb{P}^{t_{1}, t_{2}}$ onto $A^{\aleph_{0}} \times \mathcal{C} \times \mathcal{C}$. Similarly, the map

$$
\hat{\Phi}_{i}^{t}\left(\left(\tilde{x}_{1}, t\right),\left(\tilde{x}_{2}, u\right)\right)=\left(\Phi_{i}^{m}\left(\tilde{x}_{1}, \tilde{x}_{2}\right), u\right)
$$

is a homeomorphism of $\mathbb{P}_{i}^{t}$ onto $A^{\aleph_{0}} \times \mathcal{C} \times \mathcal{P}$. 
We are ready to start the argument. Since every completion of $\nu \mu_{0}^{2}$ contains a $\mathbf{G}_{\delta}$-subset $\digamma$ of $\nu \mu^{2}$ with $\nu \mu_{0}^{2} \subset \digamma$, it suffices to show that $\digamma$ contains a copy of $\mathcal{J}^{2}$. Let such an $\digamma$ be given and let $\nu \mu^{2} \backslash \digamma=\bigcup_{n} F_{n}$, where $F_{n}$ are closed in $\nu \mu^{2}$.

Select complete $\sigma$-non-archimedean bases $\mathfrak{B}_{l}=\left(\mathfrak{B}_{l}^{(1)}, \mathfrak{B}_{l}^{(2)}, \ldots\right), l=1,2,3$, in the spaces $A^{\aleph_{0}}, \mathcal{C}$ and $\mathcal{P}$, respectively. $\mathfrak{B}_{1}$ and $\mathfrak{B}_{2}$ are countable; we let $\mathfrak{B}_{1}=$ $\left\{J_{1}, J_{2}, \ldots\right\}, \mathfrak{B}_{2}=\left\{I_{1}, I_{2}, \ldots\right\}$. Letting $\mathfrak{B}^{(k)}=\left\{U \times J \times I: U \in \mathfrak{B}_{1}^{(k)}, J \in\right.$ $\left.\mathfrak{B}_{2}^{(k)}, I \in \mathfrak{B}_{3}^{(k)}\right\}$, we obtain a (complete) $\sigma$-non-archimedean base in $A^{\aleph_{0}} \times \mathcal{C} \times \mathcal{P}$.

Let $K_{n}^{t_{1}, t_{2}}=\Phi^{t_{1}, t_{2}}\left[F_{n} \cap \mathbb{P}^{t_{1}, t_{2}}\right]$. Let $Q_{i}$ be the property of closed subsets $T$ of $A^{\aleph_{0}} \times \mathfrak{C} \times \mathfrak{C}$ : "all $\left(\mathfrak{a}, c^{1}, c^{2}\right) \in T$ have the same $c^{i}$ ".

3.1. $K_{n}^{t_{1}, t_{2}}$ is $Q_{1} \vee Q_{2}$-scattered.

$K_{n}^{t_{1}, t_{2}}$ is a closed subset of $A^{\aleph_{0}} \times \mathcal{C} \times \mathcal{C}$ and since $F_{n}$ are disjoint from $\nu \mu_{0}^{2}, K_{n}^{t_{1}, t_{2}}$ is disjoint from $A^{\aleph_{0}} \times B \times B$. The rest of the proof is the standard procedure of producing the homeomorph of the Cantor set in complete dense-in-itself metric spaces. If 3.1 fails, then there is a closed subset $M$ of $K_{n}^{t_{1}, t_{2}}$ such that for every $\mathbf{U} \in \mathfrak{B}$ with $\mathbf{U} \cap M \neq \emptyset$ there are points $p, q \in \mathbf{U} \cap M$ for which both $c^{1}$ 's and $c^{2}$ 's are distinct. (Strictly speaking, we get the existence of points $p_{1}, p_{2}, q_{1}, q_{2}-$ not necessarily distinct - so that $p_{1}, p_{2}$ have distinct $c^{1}$ 's and $q_{1}, q_{2}$ have distinct $c^{2}$ 's. Patiently examining possible cases we get the stated conclusion.) Starting with an arbitrary $\mathbf{U} \in \mathfrak{B}$ with $\mathbf{U} \cap M \neq \emptyset$, we denote the above-mentioned points by $p_{0}$ and $p_{1}$. Now we select $\mathbf{U}_{0}, \mathbf{U}_{1} \in \mathfrak{B}$ such that $p_{i} \in \mathbf{U}_{i} \subset \mathbf{U}$ and so that, writing $\mathbf{U}_{i}=U_{i} \times J_{i} \times I_{i}$, we have $J_{0} \cap J_{1}=\emptyset=I_{0} \cap I_{1}$. Now we select points $p_{i, 0}, p_{i, 1} \in \mathbf{U}_{i} \cap M$ for which both $c^{1}$ 's and $c^{2}$ 's are distinct. Repeating the above - in a standard way - infinitely many times we obtain a system of members of $\mathfrak{B}$ and of points of $M$ indexed by all finite sequences of 0's and 1's. Passing to the limit, we obtain the set of points $\left(\mathfrak{a}_{\mathfrak{i}}, c_{\mathfrak{i}}^{1}, c_{\mathfrak{i}}^{2}\right)$, - the index $\mathfrak{i}$ runs through all infinite sequences of 0 's and 1 's - such that $c_{\mathfrak{i}}^{1} \neq c_{\mathfrak{i}^{\prime}}^{1}$ and $c_{\mathfrak{i}}^{2} \neq c_{\mathfrak{i}^{\prime}}^{2}$ for every two distinct $\mathfrak{i}$ and $\mathfrak{i}^{\prime}$ and the set $C=\left\{\left(c_{\mathfrak{i}}^{1}, c_{\mathfrak{i}}^{2}\right)\right\}_{\mathfrak{i}}$ is closed in $\mathcal{C} \times \mathcal{C}$. Since $M$ is closed, all the $\left(\mathfrak{a}_{\mathfrak{i}}, c_{\mathfrak{i}}^{1}, c_{\mathfrak{i}}^{2}\right)$ belong to $M$. But $C$ is an oblique perfect set, therefore there is an $\mathfrak{i}_{0}$ with $\left(c_{\mathfrak{i}_{0}}^{1}, c_{\mathfrak{i}_{0}}^{2}\right) \in B^{2}$. But then $\left(\mathfrak{a}_{\mathfrak{i}_{0}}, c_{\mathfrak{i}_{0}}^{1}, c_{\mathfrak{i}_{0}}^{2}\right) \in A^{\aleph_{0}} \times B \times B$. Contradiction.

Now apply 2.5 to get the classes $\mathfrak{F}_{n, i, k}^{t_{1}, t_{2}}$ and the sets $K_{n, i, k}^{t_{1}, t_{2}}$. Let $\hat{K}_{n, i, k}^{t_{1}, t_{2}}=$ $\left(\Phi^{m_{1}, m_{2}}\right)^{-1}\left[K_{n, i, k}^{t_{1}, t_{2}}\right]$, where $m_{i}=\operatorname{ord} t_{i} . \hat{K}_{n, i, k}^{t_{1}, t_{2}}$ is closed subset of $\left(A^{\aleph_{0}}\right)^{2}$ and since all $\left(\mathfrak{a}, c^{1}, c^{2}\right) \in K_{n, i, k}^{t_{1}, t_{2}}$ have the same $c^{i}, \hat{K}_{n, i, k}^{t_{1}, t_{2}}$ has at most one element on each line parallel to the $\left(m_{i}, i\right)$-axis.

Let $K_{n, i}^{* t}=\Phi_{i}^{t}\left[F_{n} \cap \mathbb{P}_{i}^{t}\right]$; the handling of these sets is more intricate than that of $K_{n}^{t_{1}, t_{2}}$. Let $Q$ be the property of closed subsets $T$ of $A^{\aleph_{0}} \times \mathcal{C} \times \mathcal{P}$ : "all $(\mathfrak{a}, c, u) \in T$ have the same $c "$.

3.2. $K_{n, i}^{* t}$ is Q-scattered.

The proof is similar to that of 3.1 but now, assuming that 3.2 fails, we can produce a set of points $\left(\mathfrak{a}_{\mathfrak{i}}, c_{\mathfrak{i}}, u_{\mathfrak{i}}\right) \in K_{n, i}^{* t}$ in which $c_{\mathfrak{i}}$ form a perfect set and $u_{\mathfrak{i}}$ are dyadic irrational but not necessarily distinct. Taking $\mathfrak{i}_{0}$ with $c_{\mathfrak{i}_{0}} \in B$, we have $\left(\mathfrak{a}_{\mathfrak{i}_{0}}, c_{\mathfrak{i}_{0}}, u_{\mathfrak{i}_{0}}\right) \in \nu \mu_{0}$ and we have the contradiction.

Now apply 2.5 to get the classes $\mathfrak{F}_{n, i, k}^{t}$ and the sets $K_{n, i, k}^{* t}$. Further, let

$$
\begin{gathered}
\mathfrak{F}_{n, i, k, l_{1}, l_{2}}^{t}=\left\{U \times J \times I \in \mathfrak{F}_{n, i, k}^{t}: J=J_{l_{1}}, I=I_{l_{2}}\right\}, \\
K_{n, i, k, l_{1}, l_{2}}^{* t}=K_{n, i, k}^{* t} \cap \bigcup \mathfrak{F}_{n, i, k, l_{1}, l_{2}}^{t} .
\end{gathered}
$$


Let $\overline{K_{n, i, k, l_{1}, l_{2}}^{* t}}$ be the closure of $K_{n, i, k, l_{1}, l_{2}}^{* t}$ in $A^{\aleph_{0}} \times \mathcal{C} \times \mathcal{J}$ (under the natural embedding of $\mathcal{P}$ in $\mathcal{J}$ ). Since $\mathfrak{F}_{n, i, k, l_{1}, l_{2}}^{t}$ is discrete,

$$
\overline{K_{n, i, k, l_{1}, l_{2}}^{* t}}=\bigcup\left\{\overline{K_{n, i, k}^{* t}} \cap\left(U \times J_{l_{1}} \times I_{l_{2}}\right): U \times J_{l_{1}} \times I_{l_{2}} \in \mathfrak{F}_{n, k, j}^{\prime t *}\right\}
$$

therefore all $(\mathfrak{a}, c, u) \in \overline{K_{n, i, k, l_{1}, l_{2}}^{* t}}$ still have the same $c$. Finally, let $K_{n, i, k, l_{1}, l_{2}}^{t}$ be the projection of $\overline{K_{n, i, k, l_{1}, l_{2}}^{* t}}$ onto $A^{\aleph_{0}} \times \mathcal{C}$. Since this is a projection along a compact axis, $K_{n, i, k, l_{1}, l_{2}}^{t}$ is closed in $A^{\aleph_{0}} \times \mathcal{C}$ and all $(\mathfrak{a}, c) \in \overline{K_{n, i, k, l_{1}, l_{2}}^{t}}$ still have the same c. Now, letting $\hat{K}_{n, i, k, l_{1}, l_{2}}^{t}=\left(\Phi_{i}^{m}\right)^{-1}\left[K_{n, i, k, l_{1}, l_{2}}^{t}\right]$, where $m=$ ord $t$, we obtain a closed subset of $\left(A^{\aleph_{0}}\right)^{2}$ which has at most one element on each line parallel to the $(m, i)$-axis.

We thus have a countable collection of closed subsets

$$
\hat{K}_{n, i, k}^{t_{1}, t_{2}} \quad \text { and } \quad \hat{K}_{n, i, k, l_{1}, l_{2}}^{t}
$$

of $\left(A^{\aleph_{0}}\right)^{2}$ each of which has at most one element on all lines parallel to some axis. Grouping these sets properly we obtain $\mathbf{F}_{\sigma}$-sets to which $\mathrm{S}\left(\aleph_{0}\right)$ applies. Hence there is an $\left(\tilde{x}_{0}, \tilde{y}_{0}\right) \in\left(A^{\aleph_{0}}\right)^{2}$ which does not belong to any of the sets in (1). But then, verifying the definitions of these sets we find that $\left(\left(\tilde{x}_{0}, t\right),\left(\tilde{y}_{0}, s\right)\right) \in \digamma$ for every $t_{1} ; t_{2} \in \mathcal{J}$. In other words, $\hat{h}$ maps (homeomorphically) the $\tilde{x}_{0} \tilde{y}_{0}$-plank of $\digamma$ onto $\mathcal{J}^{2}$; thus $\digamma$ has a copy of $\mathcal{J}^{2}$.

\section{Extension of 1.1: Completions of Subspaces $\nu \mu_{0}^{2}$}

Given a subset $S$ of $\mathcal{J}^{2}$ and a subspace $\kappa$ of $\nu \mu^{2}$, the $\kappa$-cylinder over $S$ is the set $\kappa(S)=\tilde{h}^{-1}[S] \cap \kappa(\operatorname{explicitly~} \kappa(S)=\{((\tilde{x}, t),(\tilde{y}, s)) \in \kappa:(t, s) \in S\})$.

4.1. If $S \subset \mathcal{J}^{2}$ is either $\mathbf{G}_{\delta}$-set or a Bernstein set in $\mathcal{J}^{2}$, then every completion of $\nu \mu_{0}(S)$ has a horizontal plank homeomorphic to a completion of $S$

Proof. The proof is the repetition of the arguments of the preceding section with some changes. 3.1 is applied only to those dyadic rationals $t_{1}, t_{2}$ for which $\left(t_{1}, t_{2}\right) \in$ $S$. 3.2 requires more attention. $\mathbb{P}_{i}^{t}$ are replaced by $\mathbb{P}_{i}^{t}(S)$ and $K_{n, i}^{* t}$ are replaced by $K_{n, i}^{* t}(S)=\Phi_{i}^{t}\left[F_{n} \cap \mathbb{P}_{i}^{t}(S)\right]$. Proof is again by contradiction; i.e., we assume that $K_{n, i}^{* t}(S)$ is not $Q$-scattered. If $S$ is $\mathbf{G}_{\delta}$-set, then we work in the space $A^{\aleph_{0}} \times \mathcal{C} \times$ $\left(S \cap \mathcal{J}_{i}^{t}\right) . \mathfrak{B}_{3}$ will now stand for complete $\sigma$-non-archimedean base in $S \cap \mathcal{J}_{i}^{t}$ (use 2.1 to get such a base) and the rest of the argument is as before.

In the case when $S$ is a Bernstein set, we work under the assumption (recall the remarks at the beginning of sect. 3 ) that $B$ is a $\mathcal{D}$-2-Bernstein, with $\mathcal{D}=\left\{D_{i}^{t}\right.$ : $t$ - dyadic rational, $i=1,2\}$, where $D_{i}^{t}$ is the intersection $\mathcal{P}_{i}^{t} \cap S$ treated as a subset of $\mathcal{P}$. As before, we work in the space $A^{\aleph_{0}} \times \mathcal{C} \times \mathcal{P}$, but we distinguish two cases. Let $\mathbf{Q}^{\prime}$ be the property of closed subsets $T$ of $A^{\aleph_{0}} \times \mathcal{C} \times \mathcal{P}$ : "all $(\mathfrak{a}, c, u) \in T$ have the same $u$ ". If $K_{n, i}^{* t}(S)$ is $\mathbf{Q}^{\prime}$-scattered, then we select a $\mathbf{U}_{0}=U_{0} \times J_{0} \times I_{0} \in \mathfrak{B}$ so that all $(\mathfrak{a}, c, u) \in K_{n, i}^{* t}(S) \cap \mathbf{U}$ have the same $u$, say $u_{0}$. We can get a set of points $\left(\mathfrak{a}_{\mathfrak{i}}, c_{\mathfrak{i}}, u_{0}\right) \in K_{n, i}^{* t}(S)$ so that $c_{\mathfrak{i}}$ form a perfect set - contradiction (same as

\footnotetext{
${ }^{2}$ In case $S$ is a Bernstein set, the set $A$ used in the definition of $\nu \mu_{0}$ depends on $S$. Thus, strictly speaking, this part of the theorem should be phrased as: if $S$ is a Bernstein set, then there exists a $\nu \mu_{0}$ so that every completion .... .
} 
in sect. 3). If $K_{n, i}^{* t}(S)$ is not $\mathbf{Q}^{\prime}$-scattered, then $K_{n, i}^{* t}(S)$ is not $\mathbf{Q} \vee \mathbf{Q}^{\prime}$-scattered; hence, working as in the proof of 3.1, we get a set of points $\left(\mathfrak{a}_{\mathfrak{i}}, c_{\mathfrak{i}}, u_{\mathfrak{i}}\right) \in K_{n, i}^{* t}(S)$ so that $\left(c_{\mathfrak{i}}, u_{\mathfrak{i}}\right)$ form an oblique perfect set $\left(u_{\mathfrak{i}}\right.$ are dyadic irrationals). Since $B$ is $\mathcal{D}$-2-Bernstein, there is a $\left(c_{\mathfrak{i}_{0}}, u_{\mathfrak{i}_{0}}\right) \in B \times D_{i}^{t}$ and the contradiction is obtained by looking at the point $\left(\mathfrak{a}_{\mathfrak{i}_{0}}, c_{\mathfrak{i}_{0}}, u_{\mathfrak{i}_{0}}\right)$.

4.2. Each $H_{\tilde{x}, \tilde{y}}$ is an intersection of clopen subsets of $\nu \mu$. Consequently, if $\digamma$ is a subspace of $\nu \mu$, then the quasicomponents of $\digamma$ are contained in its horizontal planks.

Let $\mathcal{P}_{i}, i=0,1, \ldots, 4$, stand, respectively, for ind $=0$, totally disconnected (i.e., quasicomponents are one-point sets), hereditarily disconnected (i.e., components are one-point sets), pointlike (or punctiform - i.e., no non-trivial subcontinua), and "no non-trivial arcs". It is known that for $i=0,1,2,3$ there exists a $\mathbf{G}_{\delta}$-subset of the square $\mathcal{J}^{2}$ having $\neg \mathcal{P}_{i}$ and $\mathcal{P}_{i+1}$.

4.3. Theorem. a). For $i=0,1,2,3$ there exists a metric space $M$ with ind $=0$ and such that every completion of $M$ has $\neg \mathcal{P}_{i}$, but there exists a completion of $M$ which has $\mathcal{P}_{i+1}$.

b). There exists a metric space $M$ with ind $=0$ and such that for every completion $\tilde{M}$ of $M, \tilde{M} \backslash M$ has non-trivial components and hence $\operatorname{ind}(\tilde{M} \backslash M)>0$.

Proof. In both cases we let $M=\nu \mu_{0}^{2}(S)$ for a suitable $S \subset \mathcal{J}^{2}$. For part a) we take a $\mathbf{G}_{\delta}$-subset $S$ of $\mathcal{J}^{2}$ with $\neg \mathcal{P}_{i}$ and $\mathcal{P}_{i+1}$. By 4.1 , every completion of $M$ has $\neg \mathcal{P}_{i}$, and, by $4.2, \nu \mu^{2}(S)$ is a completion of $M$ which has $\mathcal{P}_{i+1}$. For b) we take $S$ so that both $S$ and $\mathcal{J}^{2} \backslash S$ are Bernstein in $\mathcal{J}^{2}$ and apply 2.3 and 4.1.

\section{Problems}

This is a very casual preview of problems arising from the present stage of development.

We now know that there exist, in ZFC, metric spaces for which neither dim nor ind $_{c}$ can be determined in $\mathrm{ZFC} 3$ It is interesting to explore the degree of indeterminacy of the dimension functions $\operatorname{dim}$ and ind $c_{c}$ as well as relations between

\footnotetext{
${ }^{3}$ As far as I know this phenomenon has not yet been observed within non-metrizable (completely regular) spaces - of course, for non-metrizable spaces we are concerned only with dim. This is somewhat curious inasmuch as pathology of dimension functions is far better documented within arbitrary spaces than within the metric ones. On the other hand, it is known that it is very easy to define spaces for which dim has not yet been determined and it is probably very difficult to determine or perhaps cannot be determined at all. For instance, given two topologies $\tau_{1}$ and $\tau_{2}$ for a set $X, \tau_{1} \subset \tau_{2}$, and an $\tau_{2}$-closed set $A \subset X$, define the intermediate topology $\tau=\left(\tau_{1} \mid \tau_{2}, A\right)$ by the closure operation $\mathrm{cl}_{\tau}(S)=\left(\mathrm{cl}_{\tau_{1}} S \cap(X \backslash A)\right) \cup \mathrm{cl}_{\tau_{2}} S$ for every $S \subset X$. The simplest instance of this procedure is, for a given space $X$ and an arbitrary $A \subset X$, to consider $\tau=\left(\tau_{1} \mid \tau_{2}, A\right)$, where $\tau_{1}$ is the original topology of $X$ and $\tau_{2}$ is the discrete one; $X$ with this $\tau$ is denoted by $X_{A}$. Even such a primitive operation leads to serious problems concerning dim. Let $A_{1}, A_{2}, \ldots$, be subsets of the Cantor set $\mathcal{C}$ and consider the product space $\prod_{n} \mathcal{C}_{A_{n}}$. One would expect that $\prod_{n=1}^{m} \mathcal{C}_{A_{n}}$ is hereditarily strongly 0 -dimensional; however, the best I can do at the present moment is to prove that $\operatorname{dim}^{2} \mathcal{C}_{A}^{2}=0$ assuming that $A$ is not too pathological. The possibility is that $\operatorname{dim} \mathcal{C}_{A}^{2}$ might depend upon set-theoretic assumptions, but at that time I did not pursue the matters further. Now it looks more interesting; in any case, I would like to challenge the masters of covering dimension to do better than I did. The infinite product $\prod_{n} \mathcal{e}_{A_{n}}$ is probably strongly 0 -dimensional but not hereditarily. (Incidentally, the intermediate topology, as well as $\sigma$-non-archimedean bases, are discussed in the Ph.D. dissertation of my former student H. P. Tan.)
} 
these functions. One would expect that this investigation would bring formulation of new set-theoretic principles possibly far more intricate than $\mathbf{S}\left(\aleph_{0}\right)$. The most obvious (and probably hopelessly difficult) question is: does there exist a 0dimensional metric space $X$ such that for every $m, n=0,1, \ldots, \infty, n \leq M$ the statement (ind $\left.{ }_{c} X=n\right)$ and $(\operatorname{dim} X=m)$ is consistent with $Z F C$ ?

$\nu \mu_{0}$ itself raises further consistency questions. According to 1.2, both of the systems of equalities

$$
\begin{array}{ll}
\text { ind }_{c} \nu \mu_{0}=0, & \operatorname{dim} \nu \mu_{0}^{2}=1 ; \\
\text { ind }_{c} \nu \mu_{0}=1, & \operatorname{dim} \nu \mu_{0}^{2}=2 .
\end{array}
$$

are consistent with ZFC. Are the remaining systems of equalities

$$
\begin{array}{ll}
\text { ind }{ }_{c} \nu \mu_{0}=1, & \operatorname{dim} \nu \mu_{0}^{2}=1 ; \\
\text { ind }{ }_{c} \nu \mu_{0}=0, & \operatorname{dim} \nu \mu_{0}^{2}=2
\end{array}
$$

consistent with ZFC?

Irreducibility (for ease of formulation we will consider only spaces with ind $=0$ ): Kulesza [Ku1 and Ostaszewski Ost] produced (in ZFC) esoteric spaces of weight $\aleph_{1}$; such spaces are automatically irreducible. Kulesza [Ku2] has also produced (in ZFC) an irreducible esoteric space of weight $2^{\aleph_{0}}$ and obtained consistency results concerning spaces of higher weight. But it is not known whether for every $\mathfrak{m}$ with $\aleph_{1}<\mathfrak{m}<2^{\aleph_{0}}$ there is, in ZFC, an irreducible esoteric space of weight $\mathfrak{m}$.

One of the possible modifications of the irreducibility is the condition 4 that every closed subset $F$ of a space $X$ with weight $F<$ weight $X$ has a base of clopen sets (i.e., $\operatorname{Ind}_{A} X=0$ ). Some of the results of Kulesza [Ku2] refer to this type of irreducibility, and it is my understanding that he has further results in this direction.

Irreducibility can also be applied to ind ${ }_{c}$ rather than to dim (or Ind). Here an obvious question is: does there exist a space $X$ with ind ${ }_{c}>$ ind (of weight, say, $2^{\aleph_{0}}$ ) which is irreducible relative to ind ${ }_{c}$ but reducible relative to dim - i.e., for every subspace $X^{\prime}$ of $X$ with weight $X^{\prime}<$ weight $X$ we have ind ${ }_{c} X^{\prime}=0$, but there exists a subspace $X_{0}$ with weight $X_{0}<$ weight $X$ and $\operatorname{dim} X_{0}>0$ ? It is possible that the statement that $\nu \mu_{0}$ has this property is consistent, but this, of course, cannot be settled overnight.

\section{REFERENCES}

[Dou] R. Dougherty, Narrow coverings of $\omega$-ary product spaces, Annals of Pure and Applied Logic 88 (1997), 47-91. CMP 98:03

[Ku1] John Kulesza, An example in the dimension theory of metrizable spaces, Topology and its appl. 35 (1990), 109 - 120. MR 91g:54045

[Ku2] John Kulesza, Metrizable spaces where the inductive dimensions disagree, Trans. A.M.S. 318 , no. 2 . (1990), 763-781. MR 90g:54030

[M1] S. Mrówka, Further results on E-compact spaces, Acta Math. 120 (1968), 161 - 185. MR 37:2165

[M2] , Small inductive dimension of completions of metric spaces, Proc. Amer. Math. Soc. 125 (5) (1997), 1545 - 1554. MR 97i:54043

[M3] , $N$-compactness, metrizability and covering dimension, Rings of continuous functions, Marcell Dekker, Inc., New York and Basel, 1985, pp. 248 - 275. MR 86i:54034

\footnotetext{
${ }^{4}$ This could be called *-irreducibility, but since other modifications of irreducibility are likely to appear, I am reluctant to introduce firm terminology at this time.
} 
[Ost] A. Ostaszewski, A note on the Prabir Roy space, Topology Appl. 35, no. 2-3, (1990), 95-107. MR 91j:54060

[R] P. Roy, Failure of equivalence of dimension concepts for metric spaces, Bull. A.M.S. 68 (1962), 609 - 613. MR 25:5495]

Department of Mathematics, State University of New York at Buffalo, 134 Defendorf Hall, Buffalo, New York 14224

E-mail address: mrowka@acsu.buffalo.edu 